\title{
Psychological aspect of cancer: From stressor to cancer progression (Review)
}

\author{
AIHUA YUAN ${ }^{1,2}$, SHUKUI WANG ${ }^{1}$, ZONGFANG LI $^{2}$ and CHEN HUANG $^{3}$ \\ ${ }^{1}$ Department of General Surgery, Nanjing First Hospital Affiliated to Nanjing Medical University, Nanjing, Jiangsu 210006; \\ ${ }^{2}$ Department of General Surgery, Second Affiliated Hospital of Xi'an Jiaotong University Medical College, \\ Xi'an, Shaanxi 710004; ${ }^{3}$ Key Laboratory of Environment and Genes Related to Diseases of the \\ Ministry of Education, Xi'an Jiao Tong University, Xi'an, Shaanxi 710004, P.R. China
}

Received August 13, 2009; Accepted October 20, 2009

DOI: 10.3892/etm_00000003

\begin{abstract}
Substantial evidence indicates that psychological stress can influence the incidence and progression of cancers, and adequate psychotherapies are beneficial to cancer patients. Recently, the mechanisms responsible for the effects of psychological stress on cancer cells have been extensively investigated at the systemic, biochemical and molecular levels. Accumulating data indicate that the effects of psychological stress on cancer cells are mainly mediated by key stress-related mediators and their corresponding receptors in multi-fold pathways: chronic stressors act on the paraventricular nucleus and the suprachiasmatic nuclei. The effects are then transmitted through the sympathetic nervous system and the hypothalamicpituitary-adrenal axis, amplified by the unchecked release of stress-related mediators and altered behaviors. These mediators act as immunosuppressors or mitogens in the tumor microenvironment. The converging effects of psychological stress on cancer cells finally signal through receptors of the stress mediators and cytokines to activate the intracellular pro-proliferative and pro-migratory signaling pathways, and reset the molecular clock in tumor cells. Understanding these action mechanisms of psychological stress in promoting the growth and invasion of cancer cells is crucial for devising effective interventions.
\end{abstract}

\section{Contents}

1. Introduction

2. Chronic stressors act on the nervous system

3. Systemic pathways and neuroendocrine transmitters

4. Modulation of the tumor microenvironment

Correspondence to: Professor Zongfang Li, Department of General Surgery, Second Affiliated Hospital of Xi'an Jiaotong University Medical College, Xi'an, Shaanxi 710004, P.R. China

E-mail: zongfangli@yahoo.cn

Key words: psychological stress, neurotransmitters, circadian rhythms, psychotherapy, cancer
5. Converging effects on cancer cells

6. Questions and perspective

\section{Introduction}

There is a long-standing hypothesis that psychosocial factors can influence the incidence and progression of cancers, and adequate psychotherapies may be beneficial to cancer patients $(1,2)$. Clinical and epidemiological studies have also documented a prevalence of psychological stress among cancer patients and the adverse effects on cancer outcomes. However, stress-related disorders in cancer patients are not adequately assessed nor treated due to the limited knowledge of the psychological aspect of cancer and the ineffectiveness of psychotherapies $(3,4)$. Not until the recent past few years, by using in vitro animal models and human clinical perspective study approaches, have researchers begun to uncover the complex relationship between psychosocial stress and cancer progression at the systemic, biochemical and molecular levels. Accumulating data indicate that the psychological stress caused by chronic stressors is a major risk factor for cancer occurrence, growth and metastasis (5-8). Psychological stress refers to the emotional and physiological reactions experienced when an individual confronts a situation in which the adaptation demands go beyond their coping resources (9). The effects of psychological stress on cancer cells are mediated by the same key stress-related mediators and their corresponding receptors of stress response in multifold pathways $(2,6-8)$. Understanding the action mechanisms of psychosocial stress in promoting cancer progression is crucial for devising effective interventions. In this article, we briefly review the recent theory of the mechanisms involved from stressor to cancer progression.

\section{Chronic stressors act on the nervous system}

Chronic stressors first act on the central nervous system, especially the paraventricular nucleus (PVN) and the suprachiasmatic nuclei ( $\mathrm{SCN}$ ), causing an automatic process which disrupts homeostasis (2,9-12). To maintain homeostasis, two strictly controlled information-processing cascades 
precede in similar ways to properly maintain the stress response and to keep the central pacemaker in position: the sympathetic nervous system (SNS)-adrenal medullary axis and the hypothalamic-pituitary-adrenal (HPA) axis. Activation of the SNS causes increased release of norepinephrine (NEPI) from sympathetic nerve terminals, and NEPI and epinephrine (EPI) from the adrenal medulla. Activation of the HPA axis causes increased release of corticotrophin-releasing hormone (CRH) from the PVN of the hypothalamus, resulting in increased secretion of adrenocorticopic hormone (ACTH) in the anterior pituitary. ACTH, in turn, stimulates secretion of glucocorticoid (GC) hormones from the adrenal cortex. GCs modulate the activity of the HPA axis via negative feedback effects on glucocorticoid receptors (GRs) in the hippocampus. However, the information-processing cascade is out of control, and stress-related mediators can not be supressed during psychological stress, resulting in constant abnormalities in hormone levels and behavior $(2,9,10,13)$. Meanwhile, the central circadian rhythms are disrupted for the dysfunction of the central pacemaker located in the SCN $(11,12)$. Circadian rhythms are 24-h oscillations in behavior and physiology, which provide organisms a survival advantage when confronted with cyclic changes in the environment. Circadian rhythms are driven by an internal timing machine - the 'circadian clock' operated by numerous gene products in the central pacemaker as well as in most peripheral tissues. Peripheral clocks exist in almost every cell type to regulate daily changes in cell biology. To guarantee normal growth and avoid tumor formation, the timing of cell division must be under strict control. The central pacemaker controls peripheral clocks mainly through the HPA axes and SNS. GCs and melatonin (N-acetyl-5-methoxytryptamine) are in feedback control of CRH release from SCN or HPA axes. The function of the circadian pacemaker is disrupted due to two major mechanisms: loss of feedback control of $\mathrm{CRH}$ and up-regulation of the operating genes of the circadian pacemaker such as gastrin-releasing peptide (GRP) and GRP receptors (GRPRs) in SCN (Fig. 1).

\section{Systemic pathways and neuroendocrine transmitters}

The question of how psychological stress affects cancer cells has intrigued both researchers and patients. Recent studies have confirmed that the effects of psychological stress on cancer cells are mediated by key stress hormones and their corresponding receptors through similar processes observed in the stress response $(2,6-8,11-14)$. At least three systemic pathways are involved (Fig. 1): the SNS and the sympathetic-adrenal medullary axis, the HPA axis and an altered pattern of coping. First, the sympathetic fibers that descend from the brain into peripheral tissues can release a wide variety of neurotransmitters that influence cancer growth by binding to receptors on cancer cells. Some of the most significant neurotransmitters recently identified by a series of studies include cortisol, EPI, NEPI and GRP. Sympathetic signals increase the release of EPI, NEPI and GRP from the sympathetic terminal fibers or even tumor cells. Second, the sympathetic-adrenal-medullary axis and the hypothalamic-pituitary-adrenal/ovarian axis can increase the release of EPI, NEPI, cortisol, GRP, prolactin, growth hormone, melatonin, $\beta$-endorphin and enkephalin

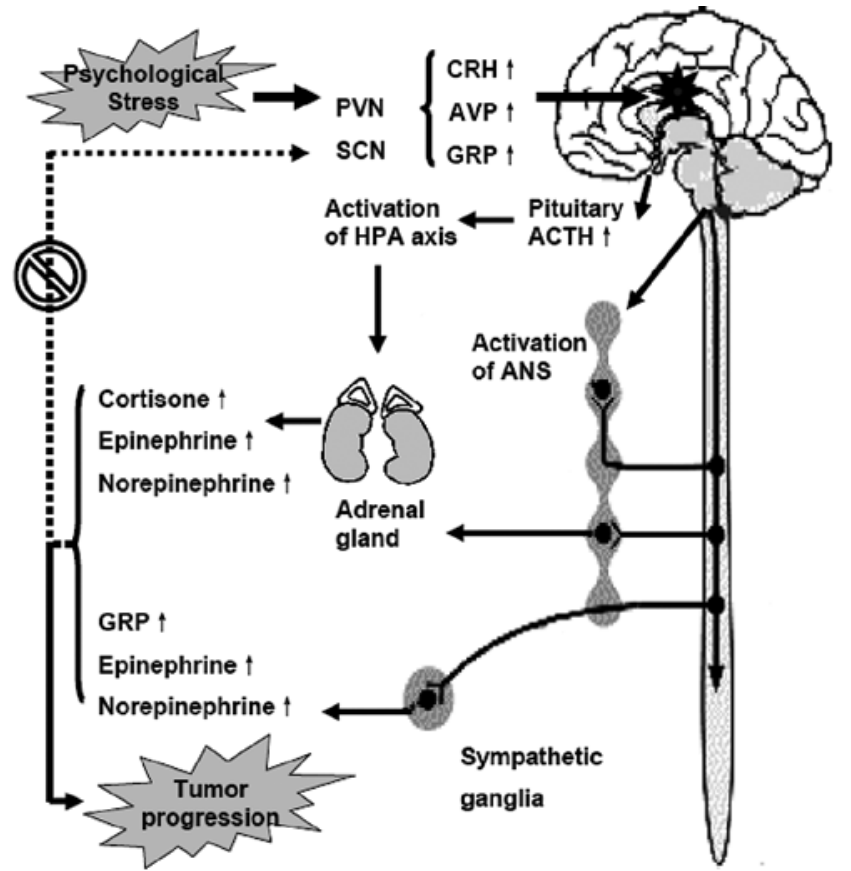

Figure 1. A schematic representation of the mechanism involved from stress to cancer progression. Chronic stressors act on the paraventricular nucleus $(\mathrm{PVN})$ and suprachiasmatic nuclei (SCN). The effects are then transmitted through the sympathetic nervous system (SNS) and the hypothalamicpituitary-adrenal (HPA) axis by checked release of stress-related mediators. Failure in suppressing the generation of stress-related mediators and disruption of the central circadian rhythms due to repeated stress response result in dysfunction of the endocrine, metabolic and immune systems, which consequently promote the progression of cancer. AVP, arginine vasopressin; ANS, autonomic nervous system (modified from refs. 2 and 11).

from the brain, pituitary and adrenal glands. The unchecked release of these stress-related neurotransmitters results in their chronic-flattened elevations in both blood and tissues. Finally, the altered pattern of coping may have an equal importance in linking stressors to cancer cells. The altered pattern of coping refers to the unhealthy behavior of individuals who suffer from psychological stress developed when coping with an adverse situation. Commonly observed unhealthy behaviors include overeating, alcoholism, sleeplessness, smoking, reduction in socialization and non-compliance to medical treatments. Through the three main pathways and numerous mediators, the effects of psychological stress are amplified and transmitted as humoral and behavioral functions to the blood, tissues and the coping mechanism.

\section{Modulation of the tumor microenvironment}

Changes in stress-related neuroendocrine transmitters during psychological stress lead to a modulation of the tumor microenvironment and immune cells. The first most important mechanism is the modulation of the immune function against tumor cells. Recent studies indicate that the impact of stress on the immune response is mediated by a bidirectional signal network between the nervous, endocrine and immune systems, and chronic stressors are associated with suppression of both cellular and humoral immune functions (Fig. 2) (12-16). For example, stress hormones affect the immune function through receptors present on immune cells, and the immune cells in 


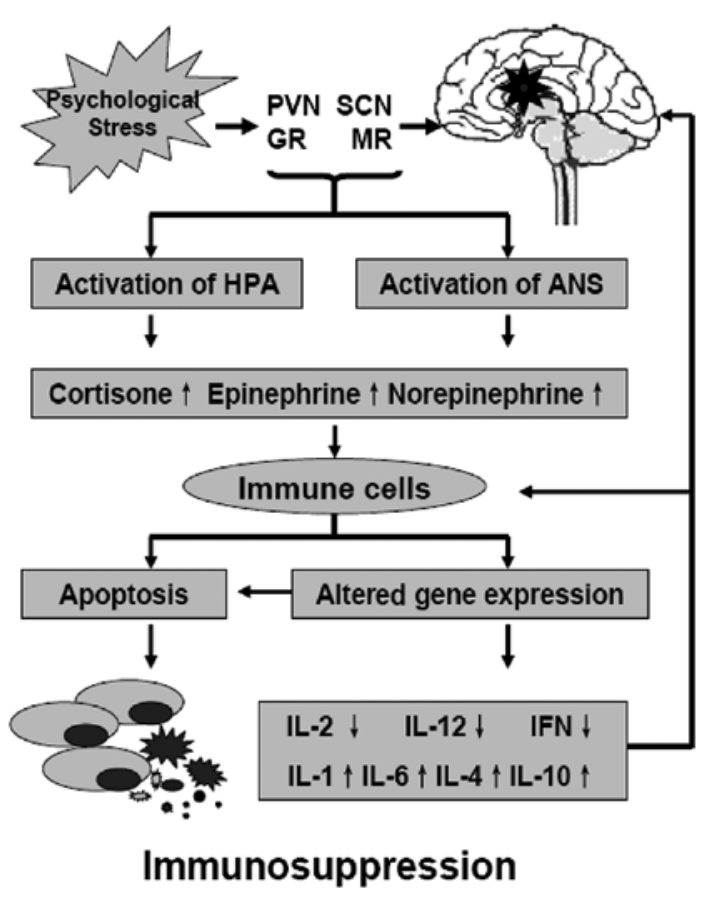

Figure 2. The deleterious effects of psychological stress on the immune system. Chronic-flattened elevations of glucocorticoids (GCs) and catecholamine activate the transcription of cytokine genes in immune cells, resulting in a shift of immune response from Th1 to Th2. This impairs both humoral and cellular immune functions against tumor cells. IL, interleukin; IFN, interferon (modified from refs. 11 and 14).

tern modulate the activity of the hypothalamus by producing cytokines. GRs expressed on a variety of immune cells, which bind cortisol interfere with the function of nuclear factor- $\kappa \mathrm{B}$ $(\mathrm{NF}-\kappa \mathrm{B})$, which regulates the activity of cytokine-producing immune cells. Adrenergic receptors (ARs) bind EPI and NEPI to activate the cAMP response element-binding protein (CREB); CREB induces the transcription of genes encoding for a variety of cytokines. Changes in gene expression result in a shift of immune response from T-helper lymphocyte type 1 cells (Th1) to T-helper lymphocyte type 2 cells (Th2), which impairs the immune responses against tumor cells (12-16). The second important mechanism is the increased generation of mitogenic factors in the tumor microenvironment. Most of the stress-related transmitters and cytokines are also mitogenic for tumor cells and can promote the growth and invasion in endocrine, paracrine and autocrine manners $(2,6-8,17)$. The third mechanism is the resettling of the tumor cell circadian clock that, decoupled from the control of central circadian rhythms via the effects of altered hormone levels on tumor cells, impacts the tumor vs. host metabolism and neuroimmune effects resulting in cancer-related immunosuppression $(11,12)$.

\section{Converging effects on cancer cells}

The total effects of psychological stress converging on cancer cells are mediated by more complicated processes, and the mechanisms remain largely unknown. However, the recognition of the direct influences of stress hormones and $\mathrm{GC}$ on cancer cells may be the most important advance in exploring the mechanisms from stressor to cancer progression.

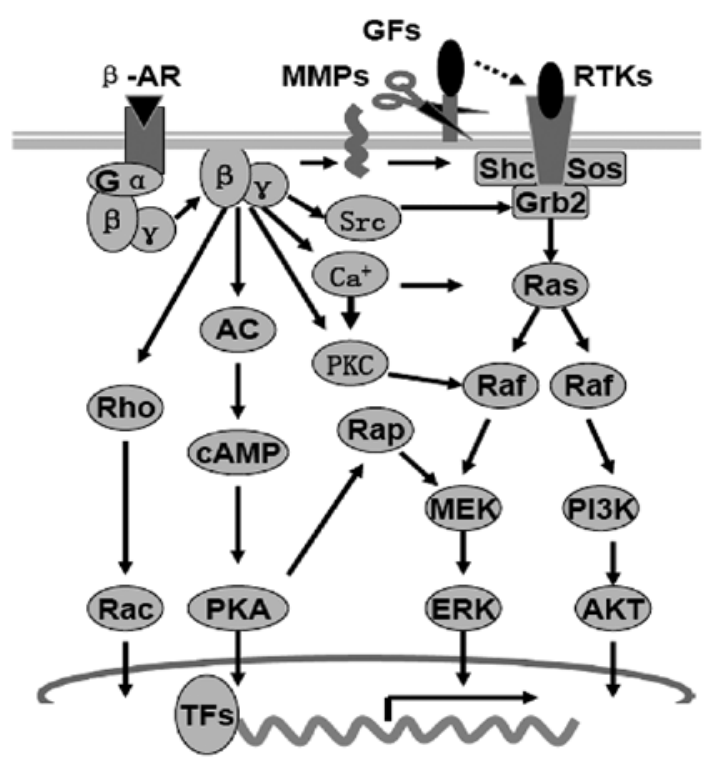

Figure 3. Known mechanisms of stress hormones in modulating the growth and metastatic potency of cancer cells. The mitogenic effect of stress hormones is mediated primarily through $\beta$-adrenergic receptors (ARs), especially $\beta 2$-AR. $\beta 2$-AR then signals either through ' $G$ protein switching' or the $\mathrm{G}$ protein-independent mechanism to activate AC/cAMP/PKA, Rho/Rac, Ras/ Raf/MEK/ERK and PI3K/AKT signal pathways in cancer cells. GFs, growth factors; TFs, transcription factors (modified from refs. 2, 11 and 19).

Using mouse model and in vitro approaches, researchers have demonstrated that the effects of psychological stress on cancer cells are mainly mediated by the key stress hormones (EPI and NEPI) and $\beta$-adrenergic receptors ( $\beta$-AR), especially the $\beta 2$-AR $(2,7,8,17)$. $\beta$-AR signals can activate several common intracellular pro-proliferative and pro-migratory signaling pathways, such as the cyclic adenosine monophosphate (cAMP)/ protein kinase A (PKA), the mitogen-activated protein kinase (MAPK)/extracellular signal-regulated kinase (ERK1/2) and phosphatidylinositol-3-kinase (PI3K)/AKT (protein kinase B) signaling pathways by (i) the ' $G$ protein switching' mechanism; (ii) the homodimerization and heterodimerization mechanism; and (iii) the MMP mechanism $(18,19)$ (Fig. 3). Activation of the cAMP-PKA signaling pathway is suggested to be the central aspect of $\beta$-AR signaling in tumor cells. Through PKA or Epac (the exchange protein directly activated by cAMP), cAMP can further activate small GTPases such as RhoA, Rac, Rap1 and Rap2 to activate MAPK/ERK1/2, PI3K/AKT and $\mathrm{Rho} / \mathrm{Rac} / \mathrm{Cdc} 42$ signaling pathways (18-24). Through homodimerization and heterodimerization with other ARs, G-protein-coupled receptors (GPCRs) or receptor tyrosine kinases (RTKs), $\beta$-AR signals directly activate the RTKs, or increase in matrix metalloproteinases (MMPs), which can release growth factors to activate the RTKs indirectly $(25,26)$.

GCs can function in synergy with stress hormones to promote cancer progression. Dexamethasone, prednisone and cortisone, for example, can promote cell survival and chemoresistance in a number of solid tumors $(27,28)$. The mechanism involves direct stimulation of cell growth and survival, as well as immunosuppression (Fig. 4). GRs are present in a number of human malignancies (29). Activated-GR acts as a transcription factor, either through 


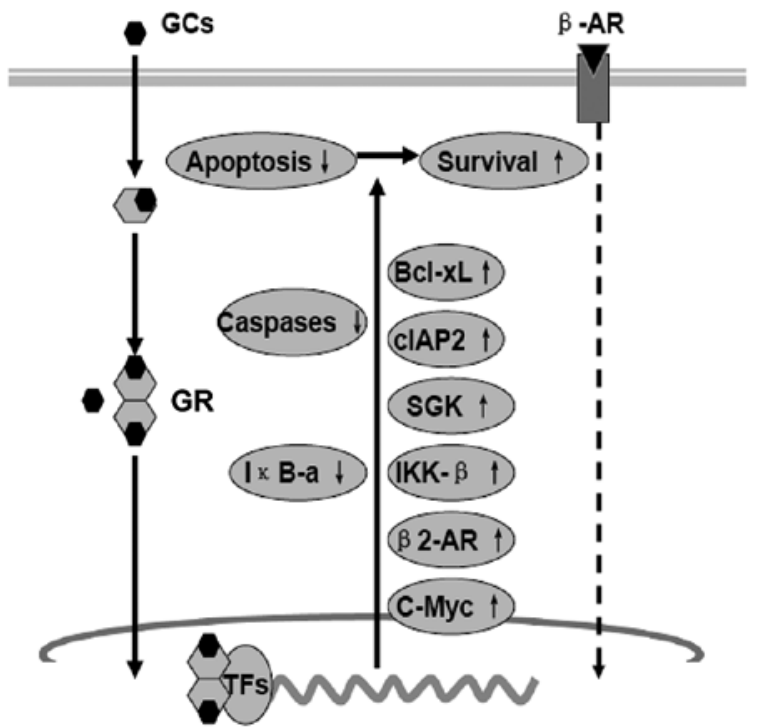

Figure 4. Glucocorticoids (GCs) work in synergy with stress hormones to support cancer cell survival.Through binding to GC response elements (GREs) and interfering with other transcription factors, GCs up-regulate c-Myc, Bcl-xL, cIAP2, SGK and $\beta 2$-adrenergic receptors (ARs) in tumor cells to promote the growth and survival of cancer cells. TFs, transcription factors (drawn by reviewing refs. 27-35).

binding to GC response elements (GRE) in the regulatory sequences of target genes or through cross-talk and/or interference with other transcription factors such as activator protein-1 (AP-1), signal transducers and activators of transcription 5 (STAT5) and NF- $\mathrm{BB}$ to promote the growth and survival of cancer cells. GCs can also support growth and metastasis of cancer cells through the up-regulation of proto-oncogene c-Myc, anti-apoptotic protein $\mathrm{Bcl}-\mathrm{xL}$, cytosolic caspase inhibitor cIAP2 and $\beta 2-\mathrm{AR}$ (30-32). Activity of NF- $\kappa \mathrm{B}$ has been shown to play an important role in preventing apoptosis of cancer cells. Activation of NF- $\mathrm{BB}$ requires activation of the $\mathrm{I} \kappa \mathrm{B}$ (inhibitor of NF- $\kappa \mathrm{B}$ ) kinase- $\beta$ (IKK- $\beta$ ). IKK- $\beta$ activation is preceded by phosphorylation by upstream kinases such as mitogen-activated protein kinase kinase-1 (MAPKK1 or MEK1), NF- $\kappa \mathrm{B}$-inducing kinase, protein kinase $\mathrm{C}(\mathrm{PKC})$ and $\mathrm{PI} 3 \mathrm{~K}$. GCs can also activate the IKK-NF- $\kappa B$ signal pathway by activation of the serum- and glucocorticoid-regulated kinase-1 (SGK1) and inactivation of the forkhead transcription factor 3a (FOXO3a) (33-35).

The changed tumor microenvironment also impinges on the internal cell timers or molecular clocks in tumor cells. The circadian clock functions in vivo as a tumor suppressor at the systemic, cellular and molecular levels (36). Ablation of SCN in mice resulted in an accelerated growth of implanted malignant tumors. Overexpression of either the circadian clock gene Per1 or Per2 in cancer cells inhibits growth and increases apoptosis (11,12,36-38). Repeated stress-response activation disrupts the circadian rhythms at both the central and peripheral levels (Figs. 1 and 5). There are several mechanisms by which circadian disruption might hasten tumor growth: the increased stress-related mediators and cytokines in the tumor microenvironment induce immunosuppression, influence the metabolic pathways and the circadian clock gene expression in tumor cells, and reduce

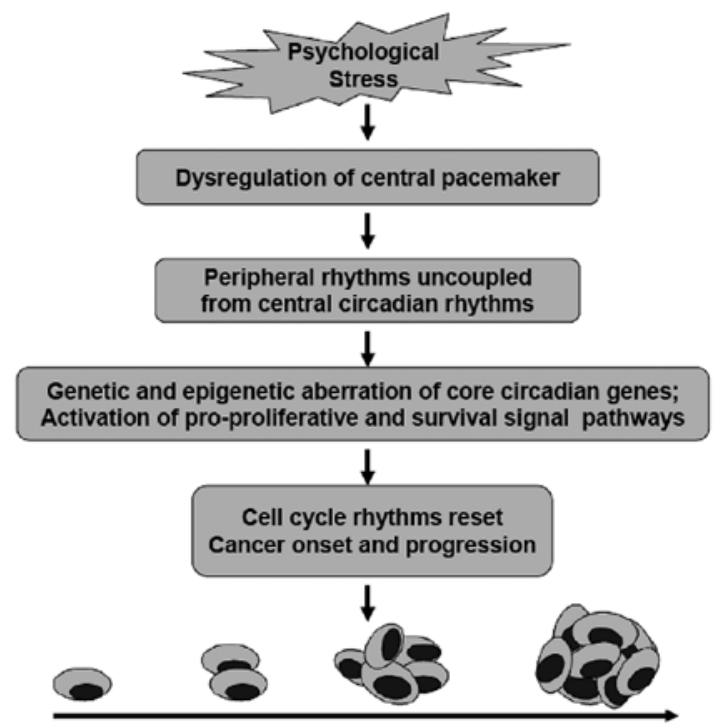

Figure 5. Mechanisms of disruption of circadian rhythms in promoting the progression of cancer: chronic stressor interferes with central circadian rhythms in both input and output pathways, leading to a dysfunction of neuroendocrine, metabolism and immunity. The increased stress-related mediators and cytokines in the tumor microenvironment induce immunosuppression, influence metabolic pathways and the circadian clock gene expression in tumor cells and reduce the sensitivity of tumor cells to treatments (modified from ref. 12).

the sensitivity of tumor cells to treatment $(11,12,39,40)$. For example, the flattened elevation of cortisone can cooperate with cell timers to establish circadian cell cycle rhythms by regulating the expression of cell cycle genes. Recent studies have revealed that expression of cell cycle genes such as Wee1, Cyclins and c-Myc is directly under the regulation of the circadian transcriptional complex (CTC). Circadian clock genes regulate cell-cycle progression and apoptosis through cAMP/PKA, MAPK/ERK1/2 and $\beta$-catenin/the T-cell factor/lymphoid enhancer binding factor (TCF/LEF) pathways $(11,12)$. Hence, the disruption of circadian rhythms, in a sense, is also a mediator of psychological stress. However, the detailed mechanisms that accounts for the effects of psychological stress on central circadian rhythms and the molecular clock remain to be clarified.

\section{Questions and perspective}

As described above, psychological stress is closely associated with tumorigenesis and cancer progression. The mechanisms for mediating the effects of psychological stress can be outlined. Chronic stressors act on the PVN and SCN. The effects are then transmitted through SNS and the HPA axis, amplified by the unchecked release of stress-related mediators and altered behaviors. All these mediators act as immunosuppressors or mitogens in the tumor microenvironment. The converging effects of psychological stress on cancer cells finally signal through receptors of the stress mediators and cytokines to activate the intracellular pro-proliferative and pro-migratory signaling pathways and reset the molecular clock in tumor cells (Figs. 1-5). There emerges an opportunity to devise effective interventions targeting the signaling pathways from stressor to cancer progression for anticancer 
treatment; for example, psychosocial and the pharmacological interventions. Recent studies have demonstrated that social support and massage therapies, especially cognitive-behavioral stress management (CBSM, a structured, manualized group intervention that emphasizes skill learning, cognitive behavioral modification and relaxation training) have potent effects of improving the outcome of cancer patients (41-43). Some drugs such as antidepressants and $\beta$-AR antagonist also exhibit certain effectiveness in attenuating the effect of psychological stress on cancer cells (44-47). However, it is not so simple; the in vivo mechanisms involved in the influence of psychological stress on cancer cells and most of the details remain unclear (48). Some questions must be resolved before devising more effective interventions. The first question involves how chronic stressors act on the PVN and SCN. This is important for devising adequate interventions to initially protect cancer patients from psychological stress. The second question must answer how many key effectors are in tumor cells. As there are several known key stress-related mediators responsible for the influence of psychological stress on cancer cells and each acts on the cell in synergy with others through different processes, it is impossible to develop one blocker for all. The third question must addres how psychological stress affects the molecular clock. Since the circadian clock functions in vivo as a tumor suppressor, it is logical to assume that the effect of psychological stress can be suppressed by the up-regulation of a certain key circadian gene. By exploring the cascades linking the psychological stress to cancer progression, some useful targets will be found for anticancer treatment in the future.

\section{References}

1. Riley V: Mouse mammary tumors: alteration of incidence as apparent function of stress. Science 189: 465-467, 1975.

2. Antoni MH, Lutgendorf SK, Cole SW, Dhabhar FS, Sephton SE, McDonald PG, Stefanek M and Sood AK: The influence of bio-behavioural factors on tumour biology: pathways and mechanisms. Nat Rev Cancer 6: 240-248, 2006.

3. Massie MJ: Prevalence of depression in patients with Cancer. J Natl Cancer Inst Monogr 32: 57-71, 2004.

4. Carlson LE, Angen M, Cullum J, Goodey E, Koopmans J, Lamont L, MacRae JH, Martin M, Pelletier G, Robinson J, Simpson JS, Speca M, Tillotson L and Bultz BD: High levels of untreated distress and fatigue in cancer patients. Br J Cancer 90: 2297-2304, 2004.

5. Garssen B: Psychological factors and cancer development: evidence after 30 years of research. Clin Psychol Rev 24: 315-338, 2004.

6. Chida Y, Hamer M, Wardle J and Steptoe A: Do stress-related psychosocial factors contribute to cancer incidence and survival? Nat Clin Pract Oncol 5: 466-475, 2008.

7. Thaker PH, LY Han, Kamat AA, Arevalo JM, Takahashi R, Lu C, Jennings NB, Armaiz-Pena G, Bankson JA, Ravoori M, Merritt WM, Lin YG, Mangala LS, Kim TJ, Coleman RL, Landen CN, Li Y, Felix E, Sanguino AM, Newman RA, Lloyd M, Gershenson DM, Kundra V, Lopez-Berestein G, Lutgendorf SK, Cole SW and Sood AK: Chronic stress promotes tumor growth and angiogenesis in a mouse model of ovarian carcinoma. Nat Med 12: 939-944, 2006.

8. Sood AK, Bhatty R, Kamat AA, Landen CN, Han L, Thaker PH, Li Y, Gershenson DM, Lutgendorf S and Cole SW: Stress hormone-mediated invasion of ovarian cancer cells. Clin Cancer Res 12: 369-375, 2006.

9. McEwen BS: Mood disorders and allostatic load. Biol Psychiatry 54: 200-207, 2003.

10. Vanitallie TB: Stress: a risk factor for serious illness. Metabolism 51: 40-45, 2002.
11. Fu L and Lee CC: The circadian clock: pacemaker and tumour suppressor. Nat Rev Cancer 3: 350-361, 2003.

12. Sephton S and Spiegel D: Circadian disruption in cancer: a neuroendocrine-immune pathway from stress to disease? Brain Behav Immun 17: 321-328, 2003.

13. Glaser R and Glaser JK: Stress-induced immune dysfunction: implications for health. Nat Rev Immunol 5: 243-251, 2005.

14. Reiche EM, Nunes SO and Morimoto HK: Stress, depression, the immune system and cancer. Lancet Oncol 5: 617-625, 2004.

15. Padgett DA and Glaser R: How stress influences the immune response. Trends Immunol 24: 444-448, 2003.

16. Segerstrom SC and Miller GE: Psychological stress and the human immune system: A meta-analytic study of 30 years of inquiry. Psychol Bull 130: 601-630, 2004.

17. Lutgendorf SK, Cole S, Costanzo E, Bradley S, Coffin J, Jabbari S, Rainwater K, Ritchie JM, Yang $M$ and Sood AK: Stress-related mediators stimulate vascular endothelial growth factor secretion by two ovarian cancer cell lines. Clin Cancer Res 9: 4514-4521, 2003.

18. Hall RA: Beta-adrenergic receptors and their interacting proteins. Semin Cell Dev Biol 15: 281-288, 2004.

19. Dorsam RT and Gutkind JS: G-protein-coupled receptors and cancer. Nat Rev Cancer 7: 79-94, 2007.

20. O'Connor KL and Mercurio AM: Protein kinase A regulates Rac and is required for the growth factor-stimulated migration of carcinoma cells. J Biol Chem 276: 47895-47900, 2001.

21. Rangarajan S, Enserink JM, Kuiperij HB, Rooij J, Price LS, Schwede F and Bos JL: Cyclic AMP induces integrin-mediated cell adhesion through Epac and Rap1 upon stimulation of the 32-adrenergic receptor. J Cell Biol 160: 487-493, 2003.

22. Schmitt JM and Stork JS: $\beta 2$-Adrenergic receptor activates extracellular signal-regulated kinases (ERKs) via the small $\mathrm{G}$ protein Rap1 and the serine/threonine kinase B-Raf. J Biol Chem 275: 25342-25350, 2000.

23. Shenoy SK, Drake MT, Nelson CD, Houtz DA, Kunhong X, Madabushi S, Reiter E, Premont RT, Lichtarge $O$ and Lefkowitz RJ: $\beta$-arrestin-dependent, $G$ protein-independent ERK1/2 activation by the $\beta 2$-adrenergic receptor. J Biol Chem 281: 1261-1273, 2006.

24. Tsurutani J, Castillo SS, Brognard J, Granville CA, Chunyu Z, Gills JJ, Sayyah J and Dennis PA: Tobacco components stimulate Akt-dependent proliferation and NF- $\kappa \mathrm{B}$-dependent survival in lung cancer cells. Carcinogenesis 26: 1182-1195, 2005.

25. Maudsley S, Pierce KL, Zamah AM, Miller WE, Ahn S, Daaka Y, Lefkowitz RJ and Luttrell LM: The $\beta 2$-Adrenergic receptor mediates extracellular signal-regulated kinase activation via assembly of a multi-receptor complex with the epidermal growth factor receptor. J Biol Chem 275: 9560-9572, 2000.

26. Drube S, Stirnweiss J, Valkova C and Liebmann C: Ligandindependent and EGF receptor-supported transactivation: lessons from beta2-adrenergic receptor signalling. Cell Signal 18: 1633-1646, 2006.

27. Herr I, Ucur E, Herzer K, Okouoyo S, Ridder R, Krammer PH, Doeberitz MK and Debatin KM: Glucocorticoid cotreatment induces apoptosis resistance toward cancer therapy in carcinomas. Cancer Res 63: 3112-3120, 2003.

28. Herr I and Pfitzenmaier J: Glucocorticoid use in prostate cancer and other solid tumours: implications for effectiveness of cytotoxic treatment and metastases. Lancet Oncol 7: 425-430, 2006.

29. NØrgaard P and Poulsen HS: Glucocorticoid receptors in human malignancies: a review. Ann Oncol 2: 541-557, 1991.

30. Petrella A, Ercolino SF, Festa M, Gentilella A, Tosco A, Conzen SD and Parente L: Dexamethasone inhibits TRAILinduced apoptosis of thyroid cancer cells via Bcl-xL induction. Eur J Cancer 42: 3287-3293, 2006.

31. Runnebaum IB and Brüning A: Glucocorticoids inhibit cell death in ovarian cancer and up-regulate caspase inhibitor cIAP2. Clin Cancer Res 11: 6325-6332, 2005.

32. Hadcock JR, Wang HY and Malbon CC: Agonist-induced destabilization of beta-adrenergic receptor mRNA. Attenuation of glucocorticoid-induced up-regulation of beta-adrenergic receptors. J Biol Chem 264: 19928-19933, 1989.

33. Landen CN Jr, Lin YG, Armaiz Pena GN, Das PD, Arevalo JM, Kamat AA, Han LY, Jennings NB, Spannuth WA, Thaker PH, Lutgendorf SK, Savary CA, Sanguino AM, Lopez-Berestein G, Cole SW and Sood AK: Neuroendocrine modulation of signal transducer and activator of transcription- 3 in ovarian cancer. Cancer Res 67: 10389-10396, 2007. 
34. Liping Z, Cui R, Xiaodong CH and Jie D: Antiapoptotic effect of serum and glucocorticoid-inducible protein kinase is mediated by novel mechanism activating IкB kinase. Cancer Res 65: 457-464, 2005.

35. Wei W, Min Z, Brickley DR, Pew $\mathrm{T}$ and Conzen SD: Glucocorticoid receptor activation signals through forkhead transcription factor 3a in breast cancer cells. Mol Endocrinol 20: 2304-2314, 2006.

36. Goodspeed MC and Lee CC: Tumor suppression and circadian function. J Biol Rhythms 22: 291-298, 2007.

37. Hastings M, O'Neill JS and Maywood ES: Circadian clocks: regulators of endocrine and metabolic rhythms. J Endocrinol 195: 187-198, 2007.

38 Chen ST, Choo KB, Hou MF, Yeh KT, Kuo SJ and Chang JG: Deregulated expression of the PER1, -PER2 and PER3 genes in breast cancers. Carcinogenesis 26: 1241-1246, 2005.

39. Rasmuson T, Ljungberg B, Grankvist K, Jacobsen J and Olsson T: Increased serum cortisol levels are associated with high tumour grade in patients with renal cell carcinoma. Acta Oncol 40: 83-87, 2001.

40. Dickmeis T: Glucocorticoids and the circadian clock. J Endocrinol 200: 3-22, 2009.

41. Andersen BL, Farrar WB, Golden-Kreutz DM, Glaser R, Emery CF, Crespin TR, Shapiro CL and Carson WE: Psychological, behavioral and immune changes after a psychological intervention: a clinical trial. J Clin Oncol 22: 3570-3580, 2004.
42. Jacobsen PB and Jim HS: Psychosocial interventions for anxiety and depression in adult cancer patients: achievements and challenges. CA Cancer J Clin 58: 214-230, 2008.

43. Küchler T, Bestmann B, Rappat S, Henne-Bruns D and WoodDauphinee S: Impact of psychotherapeutic support for patients with gastrointestinal cancer undergoing surgery: 10-year survival results of a randomized trial. J Clin Oncol 25: 2702-2708, 2007.

44. Steingart $\mathrm{AB}$ and Cotterchio $\mathrm{M}$ : Do antidepressants cause, promote, or inhibit cancers? J Clin Epidemiol 48: 1407-1412, 1995.

45. Brandes LJ: Hormetic effects of hormones, antihormones and antidepressants on cancer cell growth in culture: in vivo correlates. Crit Rev Toxicol 35: 587-592, 2005.

46. Algazi M, Plu-Bureau G, Flahault A, Dondon MG and Le MG: Is beta-blocker treatment associated with a decrease in the risk of cancer? Drug Des Discov 3: 653-661, 2006.

47. Benish M, Bartal I, Goldfarb Y, Levi1 B, Avraham1 R, Raz A and Ben-Eliyahu S: Perioperative use of $\beta$-blockers and COX-2 inhibitors may improve immune competence and reduce the risk of tumor metastasis. Ann Surg Oncol 15: 2042-2052, 2008.

48. Boesen EH and Johansen C: Impact of psychotherapy on cancer survival: time to move on? Curr Opin Oncol 20: 372-377, 2008. 